\title{
REDES DE DRENAGEM DISTRIBUTÁRIA E FORMAS DEPOSICIONAIS NO MEGALEQUE DO TAQUARI, PANTANAL: UMA ANÁLISE BASEADA NO MDE-SRTM
}

Hiran Zani

Pós-Graduação em Geociências e Meio Ambiente - Instituto de Geociências e Ciências Exatas - IGCE Campus Rio Claro - Universidade Estadual Paulista - UNESP - Av. 24-A, 1515 - Cep 13506-900, Rio Claro - SP - Brasil e-mail: hzani@dsrinpe.br Mario Luis Assine
Departamento de Geologia Aplicada - Instituto de Geociências e Ciências Exatas - IGCE -
Campus Rio Claro - Universidade Estadual Paulista - UNESP - Av. 24-A, 1515 - Cep 13506-900, Rio Claro - SP - Brasil
e-mail: assine@ @rc.unesp.br

Aguinaldo Silva

Pós-Graduação em Geociências e Meio Ambiente - Instituto de Geociências e Ciências Exatas - IGCE Campus Rio Claro - Universidade Estadual Paulista - UNESP - Av. 24-A, 1515 - Cep 13506-900, Rio Claro - SP - Brasil e-mail: aguinald_silva@yahoo.com.br

Fabrício Aníbal Corradini

Pós-Graduação em Geociências e Meio Ambiente - Instituto de Geociências e Ciências Exatas - IGCE Campus Rio Claro - Universidade Estadual Paulista - UNESP - Av. 24-A, 1515 - Cep 13506-900, Rio Claro - SP - Brasil e-mail:f_coradini@yahoo.com.br

\begin{abstract}
Resumo
Megaleques são sistemas fluviais distributários com extensão superficial da ordem de $10^{3}$ a $10^{5} \mathrm{~km}^{2}$. Tais sistemas compõem a maior parte do trato deposicional do Pantanal Mato-grossense. Dentre eles se destaca o megaleque do Taquari, com área aproximada de $49.000 \mathrm{~km}^{2}$ e superfície caracterizada pela presença de complexa rede de canais e paleocanais distributários e de formas deposicionais com geometria lobada. Análise baseada no processamento de dados SRTM foi realizada com o objetivo de mapear as redes de drenagem distributária e as formas deposicionais. Com base em modelos digitais de elevação, dez paleo-redes de drenagem distributária foram reconhecidas na superfície do megaleque do Taquari, todas elas constituindo formas deposicionais de geometria lobada altimetricamente mais altas que seu entorno. A identificação destes elementos deposicionais é passo inicial para a reconstituição de eventos de construção e abandono de lobos, bem como para a previsão de futuras mudanças no curso do rio Taquari.
\end{abstract}

Palavras-chave: megaleque fluvial, geomorfologia fluvial, modelo digital de elevação.

\begin{abstract}
Megafans are large fluvial distributary systems with areal extent varying from $10^{3}$ to $10^{5} \mathrm{~km}^{2}$. Fluvial megafans are the main depositional system in the Brazilian Pantanal wetland, being the Taquari megafan the most remarkable of them covering an area of approximately $49.000 \mathrm{~km}^{2}$. Distributary drainage networks and depositional lobes are major geomorphic features on the Taquari megafan surface. In this study, digital elevation models (DEM) based on remote sensing were used to identify and
\end{abstract}


to map these geoforms. As a result, ten depositional lobes were recognized on the fan surface, all of them distributary drainage networks topographically higher than the surrounding areas. The recognition of these drainage and depositional features is the first step to reconstruct the paleogeographic evolution and to forecast future river channel changes.

Key words: fluvial megafan, fluvial geomorphology, digital elevation model.

\section{Introdução}

Leques aluviais são depósitos sedimentares dominados por fluxos gravitacionais ou processos fluviais que, quando observados em planta, apresentam característica forma cônica (Bull, 1977). Ocorrem frequentemente associados às escarpas existentes no limite entre relevos altos e terrenos planos adjacentes (Rachocki, 1981) e apresentam extensões que variam de dezenas de metros a poucos quilômetros (Hooke, 1967).

Condições excepcionais, como alto suprimento sedimentar de origem fluvial e a existência de grande espaço de acomodação criado, podem resultar na formação de megaleques (Leeder, 1999; Horton \& DeCelles, 2001). Estes sistemas fluviais distributários ocorrem predominantemente em bacias sedimentares de foreland e possuem extensão areal da ordem de $10^{3}$ a $10^{5}$ km² (Gohain \& Parkash, 1990; Gupta, 1997; Stanistreet \& McCarthy, 1993; DeCelles \& Cavazza, 1999).

Embora não seja propriamente uma bacia do tipo foreland, a bacia sedimentar do Pantanal possui um trato deposicional composto por vários megaleques fluviais (Figura 1), dentre os quais o megaleque do Taquari constitui a feição geomórfica mais notável da planície, com cerca de 49.000km² (Braun, 1977; Assine \& Soares, 2004).

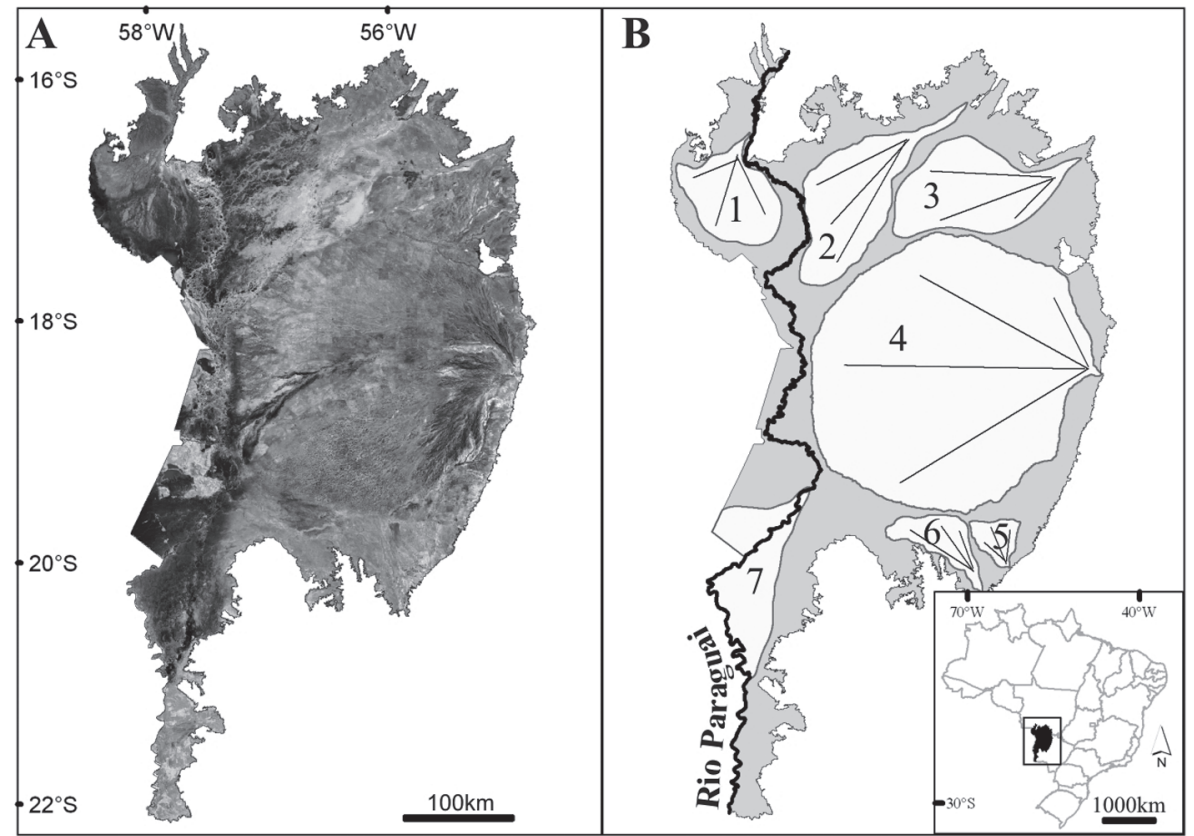

Figura 1 - Localização do Pantanal brasileiro. A) mosaico Landsat 4/5 Geocover 1990, composição R7G4B2; B) megaleques fluviais que compõe o trato deposicional da planície: 1 -Paraguai; 2 - Cuiabá; 3 -São Lourenço; 4 -Taquari; 5 -Taboco; 6 -Aquidauana e 7 Nabileque.

Uma vasta rede de canais e paleocanais distributários caracterizam a morfologia superficial do megaleque, sendo a identificação e o mapeamento destes elementos um dos meios para o reconhecimento dos processos deposicionais que atuaram em sua morfogênese (e.g. Assine, 2003). Os processos responsáveis pela formação dos megaleques estão ativos desde o Pleistoceno tardio (Ab'Saber, 1988) e sua compre- ensão é fundamental para a reconstituição da evolução geomorfológica regional e antevisão de tendências futuras de mudanças na paisagem.

Este trabalho tem como escopo reconhecer e caracterizar as redes de canais e paleocanais distributárias existentes no megaleque do Taquari, assim como identificar as principais formas que delineiam os lobos deposicionais. 


\section{Sedimentação em Leques Aluviais}

A dinâmica sedimentar em megaleques fluviais apresenta muitas semelhanças, em termos de evolução, com a dos leques aluviais dominados por fluxos de gravidade (e.g. Schumm, 1977; Leeder, 1999; Horton \& DeCelles, 2001; Assine, 2003). A análise dos trabalhos publicados sobre o tema revela a existência de modelo qualitativo de grande aplicação, tanto para leques dominados por fluxos de gravidade quanto para leques dominados por rios, baseados na existência de lobos deposicionais, ativos e abandonados (Denny, 1967; Bull, 1977; Schumm, 1977; Rachocki, 1981). Lobos deposicionais são formas alongadas que expressam o padrão de sedimentação produzido por rede de drena- gem distributária, numa dinâmica sedimentar de construção e abandono de lobos (Figura 2).

Neste ambiente de baixas declividades, a continuidade de processos de agradação e progradação sedimentar resulta em formas que apresentam cotas altimétricas superiores às das áreas adjacentes (Assine, 2005). Portanto, os principais elementos geomórficos que compõem um leque, como lobos deposicionais e complexos de canais distributários e seus diques marginais, possuem expressões altimétricas características (Volker et al., 2007). Assim, modelos digitais de elevação (MDE) constituem excelente ferramenta para o mapeamento destas formas deposicionais e para o reconhecimento de relações morfológicas, tais como superposição e truncamento dos elementos identificados.

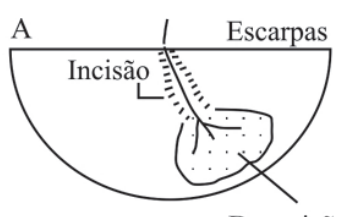

Deposição

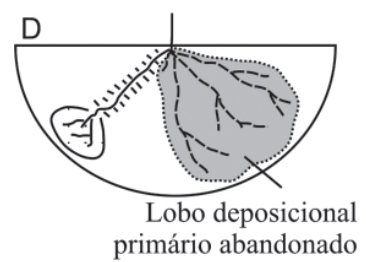

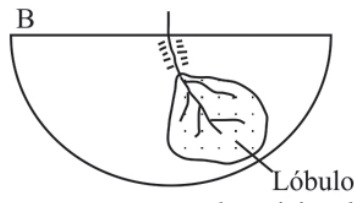

deposicional

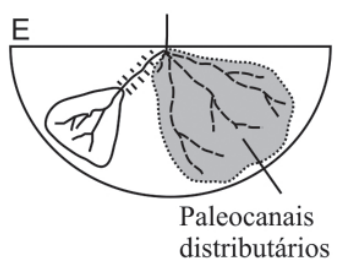

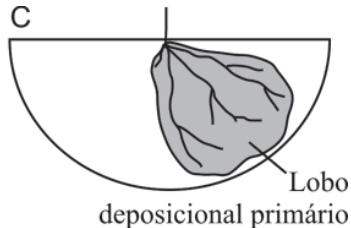

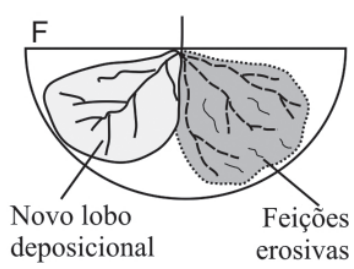

Figura 2 - Modelo de sedimentação em leques aluviais. A) incisão e entrincheiramento da zona proximal e início da deposição na zona distal; B) preenchimento sedimentar do canal à montante e progradação sedimentar para jusante; $C$ ) preenchimento do canal e aumento da rede distributária; $D$ ) abandono do lobo primário com incisão à montante do novo canal e deposição na zona distal; E) $F$ ) progradação sedimentar, aumento da rede distributária e formação de um novo lobo deposicional. Adaptado de Denny (1967), Bull (1977), Schumm (1977) e Rachocki (1981).

\section{Material e Métodos}

Os métodos utilizados neste trabalho foram exclusivamente baseados nos dados orbitais coletados pela Shuttle Radar Topographic Mission (SRTM). Esta missão teve como objetivo produzir um MDE global com resolução espacial de $\sim 30 \mathrm{~m}$ para os EUA e $\sim 90 \mathrm{~m}$ para o restante do mundo. Vários pesquisadores aplicaram com sucesso o MDE-SRTM em estudos de geomorfologia (e.g. Verstraeten, 2006; Grohmann et al., 2007; Rossetti \& Valeriano, 2007; Ehsani \& Quiel, 2008; Rossetti et al., 2008; Reinhard et al., 2008). Características detalhadas e especificidades técnicas da missão, fundamentais para a boa interpretação dos dados, podem ser encontradas em van Zyl (2001) e Far et al. (2007).

$\mathrm{O}$ procedimento inicial para o reconhecimento das diferenças altimétricas locais, que caracterizam os elementos geomórficos do megaleque, consistiu no cálculo de superfícies de tendência através de um algoritmo polinomial genérico, com a altitude $(Z)$ em função das coordenadas geográficas $(X, Y)$ :

$$
Z=\sum_{i=0}^{N} \sum_{j=0}^{N} d_{i j} X^{i} Y^{j}
$$

onde: $N$ é o grau polinômio; $i$ e $j$ são variáveis de interação da equação e $d$ o coeficiente de regressão.

Testes estatísticos paramétricos foram realizados para identificar a razão das variâncias (Teste de Fisher) entre as superfícies de tendências computadas com o MDE-SRTM original, a fim de para verificar qual é a ordem da Equação 1 que possui representatividade mínima dos dados SRTM para o megaleque do Taquari. 
A superfície de tendência calculada pela Equação 1 $\left(Z_{T R D}\right)$ e selecionada a partir do teste paramétrico foi subtraída do MDE-SRTM original $\left(Z_{S R T M}\right)$, resultando valores altimétricos relativos $\left(\mathrm{Z}_{\mathrm{RES}}\right)$, expressos por valores positivos (acima da tendência topográfica) e negativos (abaixo da tendência topográfica):

$$
Z_{\text {RES }}=Z_{\text {TRD }}-Z_{\text {SRTM }}
$$

Para a confecção do plano de informação final aplicou-se o algoritmo da curvatura mínima (Smith \& Wessel, 1990) na interpolação dos resultados da Equação 2. O MDESRTM processado foi inserido em um banco de dados geográficos (SIG) para a visualização e interpretação dos resul- tados. Finalmente, foram efetuadas análises estatísticas descritivas para a construção de gráficos e caracterização das distribuições dos modelos gerados.

\section{Resultados e Discussão}

Superfícies de tendência de graus 1 a 4 foram obtidas a partir da aplicação da Equação 1 no MDE-SRTM original (Figura 3). Os testes paramétricos de razão entre as variâncias apontaram a superfície cúbica como grau mínimo para representar a tendência topográfica do megaleque (Tabela 1).

Tabela 1 - Resultado do teste de razão entre variâncias, com 95\% de confiança.

\begin{tabular}{cccc}
\hline Grau da superfície & Ajuste dos dados $\left(\mathbf{r}^{\mathbf{2}}\right)$ & Valor F observado $^{*}$ & Hipótese aceita $^{* *}$ \\
\hline $1^{\mathbf{0}}$ & 0.82 & 1.091 & $\mathrm{H}_{\mathrm{a}}$ \\
$2^{\mathrm{o}}$ & 0.95 & 1.049 & $\mathrm{H}_{\mathrm{a}}$ \\
$3^{\mathrm{o}}$ & 0.97 & 0.996 & $\mathrm{H}_{0}$ \\
$4^{\mathrm{o}}$ & 0.97 & 0.992 & $\mathrm{H}_{0}$ \\
\hline
\end{tabular}

${ }^{*}$ Valor crítico de $F: 1.018$

${ }^{* *} H_{0}$ : a variância da superfície de tendência é igual a dos dados originais

$H_{a}:$ a variância da superfície de tendência difere dos dados originais
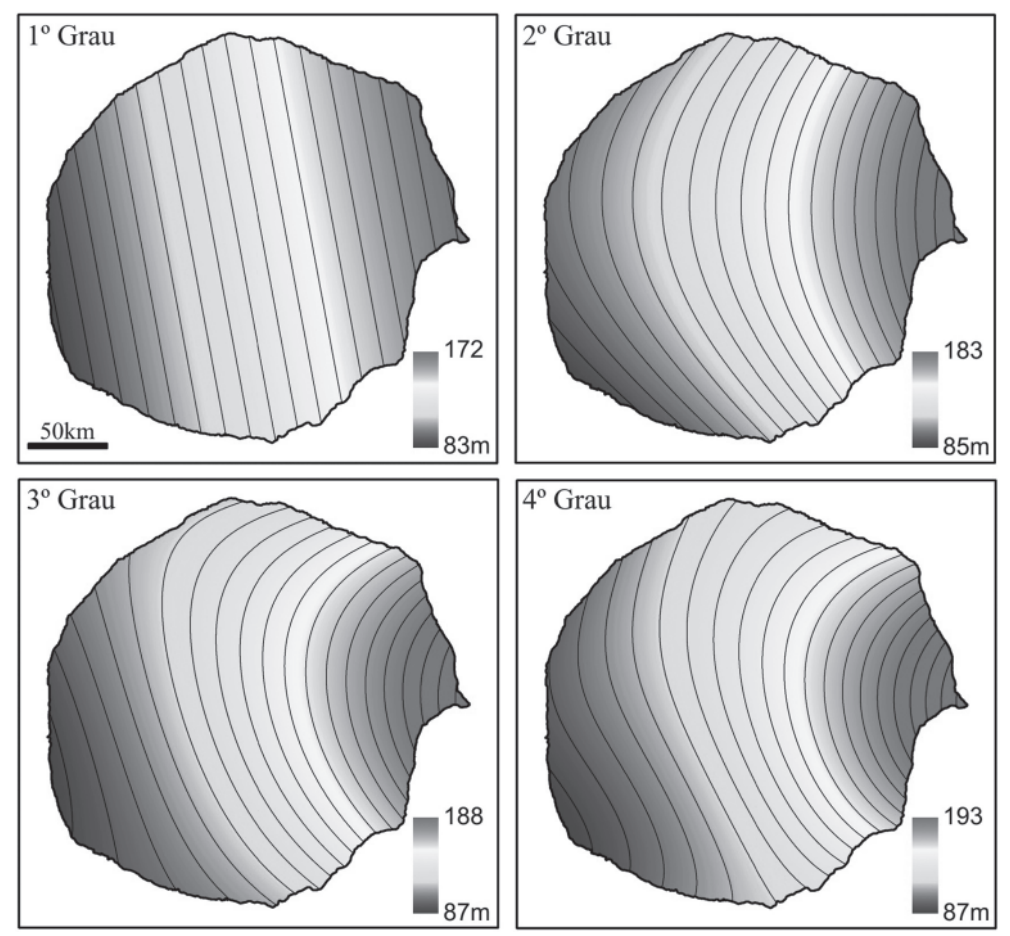

Figura 3 - Superfícies de tendência geradas para o megaleque do Taquari. 
As superfícies de tendência representam em diferentes graus de complexidade o arranjo global da topografia do megaleque do Taquari. A partir da superfície de $2^{\circ}$ grau, observa-se que isolinhas altimétricas semicirculares irradiam das altitudes mais elevadas. Este tipo de configuração topográfica é característico de leques subaéreos, de variadas dimensões, desde pequenos leques dominados por fluxos de gravidade (Bull, 1977) a megaleques fluviais com centenas de quilômetros de extensão (Gohain \& Parkash, 1990). O mesmo padrão pode ser observado em outros sistemas deposicionais distributários, como deltas (Schumm, 1977) e leques tubidíticos em plataformas continentais (Booth et al., 2003).

MDE-SRTM original
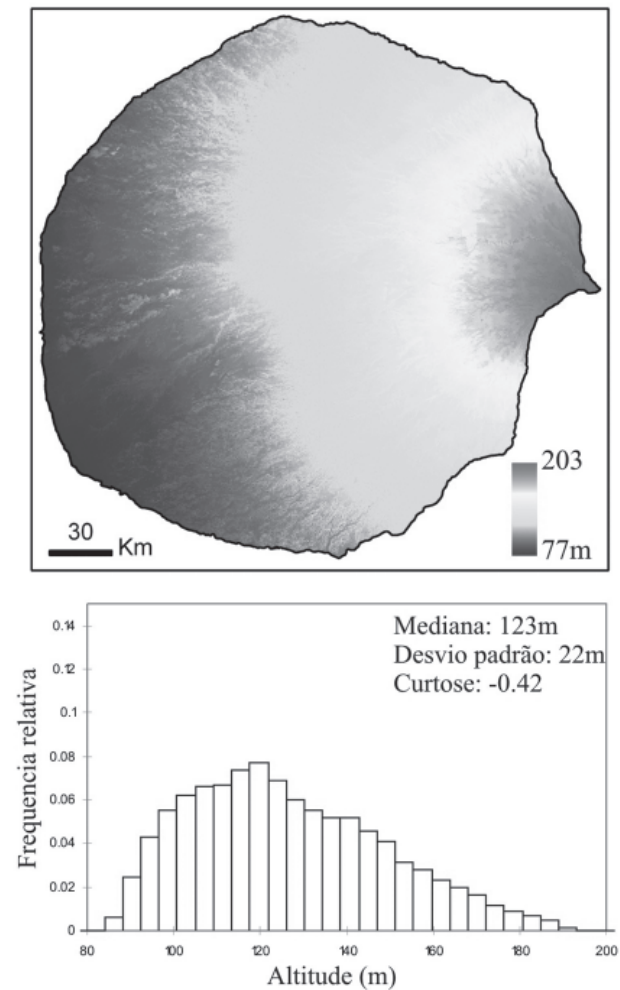

Os testes estatísticos apontaram que a superfície cúbica da Figura 3 é a que apresentou a complexidade espacial mínima para representar a topografia do megaleque. Por isso, foi subtraída da grade altimétrica original (Equação 2), resultando em novo MDE com maior contraste e menor variabilidade altimétrica com relação ao MDE-SRTM original (Figura 4), o que constituiu a base para a identificação das formas deposicionais recentes. O processamento realizado alterou o datum global, geóide WGS84, para uma superfície cúbica calculada pela Equação 1 .

\section{MDE-SRTM processado}
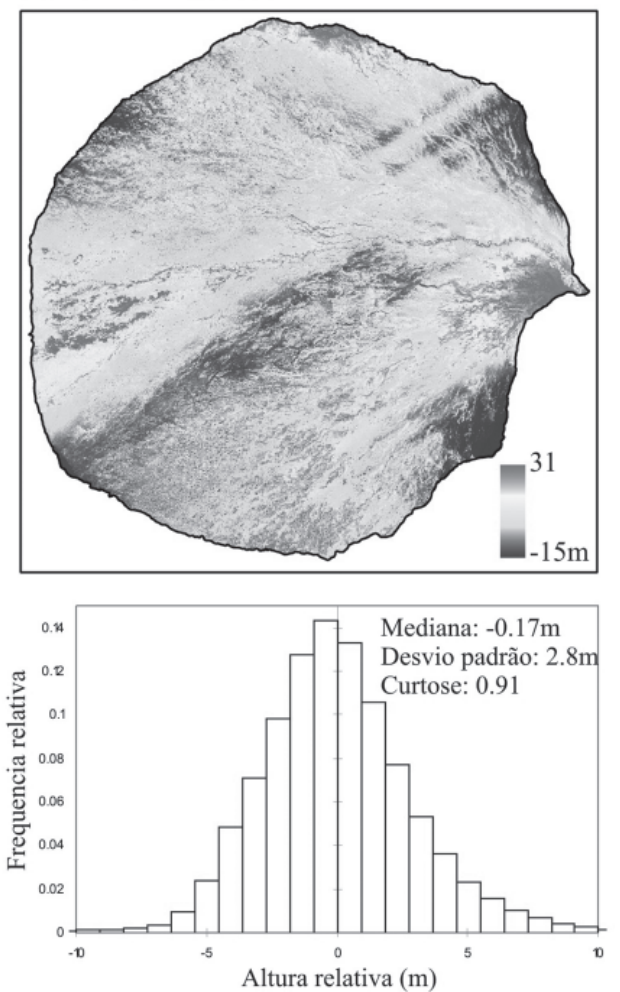

Figura 4 - Comparação entre MDE-SRTM original e processado. Respectivos histogramas demonstram que o MDE-SRTM processado possui maior frequência relativa, o que permite melhor distinção das formas deposicionais.

O MDE-SRTM processado foi fatiado em três classes de altura: A) -16 a -1m para as superfícies abaixo da tendência do megaleque; B) 0 a $2 \mathrm{~m}$ para as superfícies concordantes com a tendência e C) 3 a $32 \mathrm{~m}$ para as superfícies com alturas superiores à tendência geral (Figura 5-A). Foram identificadas dez formas deposicionais com geometria lobada, sendo cinco localizadas no cone de sedimentação atual do Taquari e cinco abandonadas (Figura 5-B)
Verificou-se que as características dos padrões de canal dos lobos antigos diferem daqueles situados no lobo distributário atual. As formas interpretadas como antigas também se mostraram consideravelmente de maior dimensão em área que as atuais. Conforme demonstrou Harvey (2002), leques aluviais registram em sua superfície modificações ocorridas nos sistemas fluviais. Alterações hidráulicas nos rios, por sua vez, podem estar associadas com mudanças climáticas recentes (e.g. Carignano, 1999; Sohn et al., 2007). 
No ambiente deposicional do megaleque, a posição altimétrica superior dos lobos deposicionais sugere que estas feições sedimentares estão sobrepostas a formas mais antigas. Assim, buscando uma ordem cronológica relativa, as formas mapeadas através do MDE-SRTM processado podem ser consideradas elementos geomórficos mais recentes na Bacia do Pantanal. Relações superficiais de truncamento entre os lobos, como pode ser observado na porção norte da Figura 5-A, também podem auxiliar na hierarquização temporal dos processos (e.g. Denny, 1967; Assine, 2003).
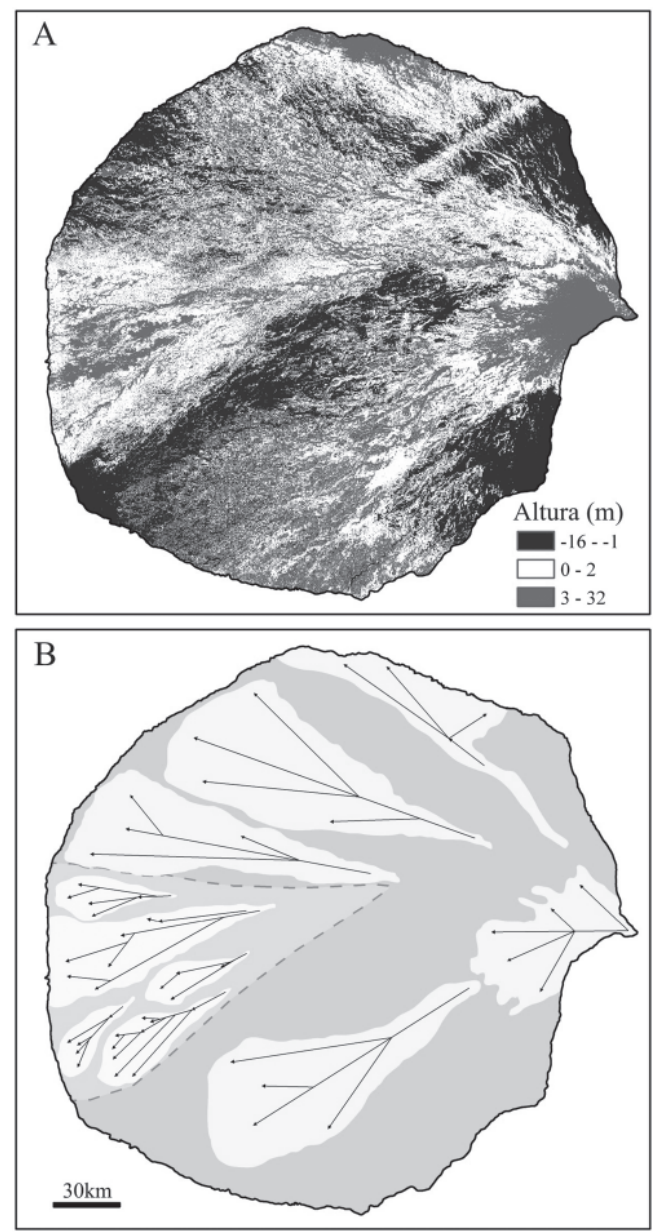

Figura 5 - Formas deposicionais identificadas. A) MDE-SRTM processado fatiado em 3 classes de altura; B)lobos deposicionais recentes (mais claro) e lobos abandonados (mais escuro).

No entanto, em áreas de baixas amplitudes altimétricas, como no Pantanal, a influência dos dosséis arbóreos pode criar formas artificiais de relevo no MDESRTM e superestimar a altitude em áreas com maior densi- dade de vegetação (Valeriano \& Abdon, 2007). Por outro lado, o tipo de vegetação existente está diretamente relacionado com a geomorfologia local (Hupp \& Osterkamp, 1985; Hupp \& Osterkamp, 1996) e pode ser utilizado como um bom indicador das formas fluviais quaternárias (e.g. Casco et al. 2005; Baroni et al., 2007). Outros elementos decorrentes de erros intrínsecos do MDE-SRTM são feições lineares com ganho altimétrico, orientadas $45^{\circ} \mathrm{NE}$, que podem ser observadas na região nordeste do MDE-SRTM processado (Figura 5-A) e não expressam componentes fisiográficos ou geomorfológicos.

A acurácia do produto altimétrico utilizado também deve ser considerada na interpretação dos resultados. Segundo Rodriguez et al. (2006) o MDE-SRTM possui precisão vertical absoluta de $\pm 9 \mathrm{~m}$, sendo que em áreas de baixas declividades como planícies fluviais, este erro diminui para $\pm 6 \mathrm{~m}$ (com $90 \%$ de intervalo de confiança). As formas delineadas no presente trabalho estão além deste limiar e, portanto, representam elementos com expressão altimétrica que compõe a paisagem.

\section{Conclusões}

Diante do exposto, conclui-se que a superfície do megaleque do Taquari é caracterizada pela presença de lobos deposicionais. Estes elementos deposicionais, morfologicamente mais altos que seu entorno, apresentam densas redes de drenagem distributária e podem ser considerados como sendo as feições mais recentes desta porção da Bacia do Pantanal.

O modelo de evolução por meio da construção e abandono de lobos deposicionais também pode ser aplicado ao megaleque do Taquari. No entanto, a configuração espacial das formas identificadas sugere uma dinâmica mais complexa e menos linear, onde formas deposicionais de diferentes idades se sobrepõem, resultando numa intrincada malha de redes superpostas de canais e paleocanais distributários de diferentes idades, numa configuração mais complexa que a sucessão proposta anteriormente por Assine (2003) e Zani et al. (2006).

\section{Agradecimentos}

Os autores externam seus agradecimentos à FAPESP pela concessão de bolsa de mestrado a Hiran Zani (06/023818) e pelo apoio às pesquisas no Pantanal Mato-Grossense (processo 07/55987-3), ao CNPq pela concessão de bolsa PQ a Mario L. Assine (308724/2006-2) e bolsa de doutorado a Aguinaldo Silva e à CAPES pela concessão de bolsa de doutorado a Fabrício Aníbal Coradini. 


\section{Referências Bibliográficas}

Ab'Saber, A.N. (1988) O Pantanal Matogrossense e a teoria dos refúgios. Revista Brasileira de Geografia, 50(2):9-57.

Assine, M.L. (2003) Sedimentação na bacia do pantanal matogrossense, centro oeste do Brasil. Tese de Livre Docência. Universidade Estadual Paulista, Rio Claro. 105p.

Assine, M.L. (2005) River avulsions on the Taquari megafan, Pantanal Wetland, Brazil. Geomorphology, 70(34): 357-371.

Assine, M.L. \& Soares, P.C. (2004) Quaternary of the Pantanal, west-central Brazil. Quaternary International, 114(1):23-34.

Baroni, C., Armiraglio, S., Gentili, R., Carton, A. (2007) Landform-vegetation units for investigating the dynamics and geomorphologic evolution of alpine composite debris cones (Valle dell' Avio, Adamello Group, Italy). Geomorphology, 84(1-2):59-79.

Booth, J.R.; Dean, M.C.; DuVernay, A.E.; Styzen, M.J. (2003) Paleo-bathymetric controls on the stratigraphic architecture and reservoir development of confined fans in the Auger Basin: central Gulf of Mexico slope. Marine and Petroleum Geology, 20(68):563-586.

Braun, E.W.G. (1977) Cone aluvial do Taquari, unidade geomórfica marcante na planície quaternária do Pantanal. Revista Brasileira de Geografia, 39(4):164-180.

Bull, W.B. (1977) The alluvial-fan environment. Progress in Physical Geography, 1(2):222-270.

Carignano, C.A. (1999) Late Pleistocene to recent climate change in Córdoba Province, Argentina: Geomorphological evidence. Quaternary International, 57-58(1):117-134.

Casco, S.L.; Chiozzi, N.I.; Neiif, J.J. (2005) La vegetación como indicador de la geomorfologia fluvial. Revista Brasileira de Geomorfologia, 6(1):123-136.

DeCelles, P.G. \& Cavazza, W. (1999) A comparison of fluvial megafans in the Cordilleran (Upper Cretaceous) and modern Himalayan foreland basin systems. Geological Society American Bulletin, 111(9):1315-1334.

Denny, C.S. (1967) Fans and pediments. American Journal of Science, 265(2):81-105.
Ehsani, A.H. \& Quiel, F. (2008) Application of Self Organizing Map and SRTM data to characterize yardangs in the Lut desert, Iran. Remote Sensing of Environment, 112(7):3284-3294.

Farr, T.G.; Rosen, P.A.; Caro, E.; Crippen, R.; Duren, R.; Hensley, S.; Kobrick, M.; Paller, M.; Rodriguez, E.; Roth, L.; Seal, D.; Shaffer, S.; Shimada, J.; Umland, J.; Werner, M.; Oskin, M.; Burbank, D.; Alsdorf, D. (2007) The Shuttle Radar Topography Mission. Review in Geophysics, 45(2):21-35.

Gohain, K. \& Parkash, B. (1990) Morphology of the Kosi megafan. In: A.H. Rachocki and M. Church (Eds.) Alluvial Fans - A field Approach. Wiley. pp151-178.

Grohmann, C.H.; Riccomini, C.; Alves, F.M. (2007) SRTMbased morphotectonic analysis of the Poços de Caldas Alkaline Massif, southeastern Brazil. Computers \& Geosciences, 33(1):10-19.

Gupta, S. (1997) Himalayan drainage patterns and the origin of fluvial megafans in the Ganges foreland basin. Geology, 25(1):11-14.

Harvey, A.M. (2002) The role of base-level change in the dissection of alluvial fans: case studies from southeast Spain and Nevada. Geomorphology, 45(1-2):67-87.

Hooke, R.L. (1967) Processes on arid-region alluvial fans. Journal of Geology, 75(4):438-460.

Horton, B.K. \& DeCelles, P.G. (2001) Modern and ancient fluvial megafans in the foreland basin system of the central Andes, southern Bolivia: Implications for drainage network evolution in fold-thrust belts. Basin Research, 13(1):43-61.

Hupp, C.R. \& Osterkamp, W.R. (1985) Bottomland vegetation distribution along Passage Creek, Virginia, in relation to fluvial landforms. Ecology, 66(3):670-681.

Hupp, C.R. \& Osterkamp, W.R. (1996) Riparian vegetation and fluvial geomorphic processes. Geomorphology 14(4):277-295.

Leeder, M. (1999) Sedimentology and Sedimentary Basins: From Turbulence to Tectonics. Blackwell. 608p.

Rachocki, A. (1981) Alluvial fans: an attempt at an empirical approach. Wiley. 161p.

Reinhard, C.; Wünnemann, B.; Krivonogov, S.K.(2008) Geomorphological evidence for the Late Holocene evolution and the Holocene lake level maximum of the Aral Sea. Geomorphology, 93(3-4):302-315. 
Rodriguez, E.; Morris, C.S.; Belz, J.E. (2006) A global assessment of the SRTM performance. Photogrammetric Engineering and Remote Sensing, 72(3):249-260.

Rossetti, D. \& Valeriano, M.M. (2007) Evolution of the lowest amazon basin modeled from the integration of geological and SRTM topographic data. CATENA, 70(2):253-265.

Rossetti, D.; Góes, A.M.; Valeriano, M.M.; Miranda, M.C. (2008) Quaternary tectonics in a passive margin: Marajó Island, northern Brazil. Journal of Quaternary Science, 23(2):121-135.

Schumm, S.A. (1977) The Fluvial System. Wiley. 338p.

Smith, W.H.F. \& Wessel, P. (1990) Gridding with Continuous Curvature Splines in Tension. Geophysics, 55(3):293-305.

Sohn, M.F.; Mahan, S.A.; Knott, J.R.; Bowman, D.D. (2007) Luminescence ages for alluvial-fan deposits in Southern Death Valley: Implications for climatedriven sedimentation along a tectonically active mountain front. Quaternary International, 166(1):49-60.

Stanistreet, I.G. \& McCarthy, T.S. (1993) The Okavango Fan and the classification of subaerial fan systems. Sedimentary Geology, 85(14):115 - 133.
Valeriano, M.M. \& Abdon, M.M.A. (2007) Aplicação de dados SRTM a estudos do Pantanal. Revista Brasileira de Cartografia, 59(1):63-71.

Verstraeten, G. (2006) Regional scale modelling of hillslope sediment delivery with SRTM elevation data. Geomorphology, 81(1-2):128-140.

Volker, H.X.; Wasklewicz, T.A.; Ellis, M.A. (2007) A topographic fingerprint to distinguish alluvial fan formative processes. Geomorphology, 88(12):34-45.

Zani, H.; Assine, M.L.; Araújo, B.C.; Merino, E.R.; Silva, A.; Fancicani, E. (2006) Lobos deposicionais na evolução do megaleque do rio Taquari, Pantanal Mato-Grossense. In: Simpósio de Geotecnologias no Pantanal, 1., Campo Grande. Anais... São José dos Campos: INPE. p. 285-292. Disponível em: < http://mtc-m17.sid.inpe.br/col/sid.inpe.br/mtcm17@80/2006/12.12.13.12/doc/p101.pdf >. Acesso em: 15 de abril de 2008.

van Zyl, J.J. (2001) The shuttle radar topography mission (SRTM): a breakthrough in remote sensing of topography. Acta Astronautica, 48(5-12):559-565. 